\title{
The number of distinct part sizes of some multiplicity in compositions of an Integer. A probabilistic Analysis
}

\author{
Guy Louchard
}

Université Libre de Bruxelles, Département d'Informatique, CP 212, Boulevard du Triomphe, B-1050 Bruxelles, Belgium

louchard@ulb.ac.be

\begin{abstract}
Random compositions of integers are used as theoretical models for many applications. The degree of distinctness of a composition is a natural and important parameter. A possible measure of distinctness is the number $X$ of distinct parts (or components). This parameter has been analyzed in several papers. In this article we consider a variant of the distinctness: the number $X(m)$ of distinct parts of multiplicity $m$ that we call the $m$-distinctness. A first motivation is a question asked by Wilf for random compositions: what is the asymptotic value of the probability that a randomly chosen part size in a random composition of an integer $v$ has multiplicity $m$. This is related to $\mathbb{E}(X(m))$, which has been analyzed by Hitczenko, Rousseau and Savage. Here, we investigate, from a probabilistic point of view, the first full part, the maximum part size and the distribution of $X(m)$. We obtain asymptotically, as $v \rightarrow \infty$, the moments and an expression for a continuous distribution $\varphi$, the (discrete) distribution of $X(m, v)$ being computable from $\varphi$.
\end{abstract}

Keywords: Mellin transforms, urns models, Poissonization, saddle point method, generating functions

\section{Introduction}

Let us fi rst recall some well-known results. Let us consider the composition of an integer $v$, i.e. $v=$ $\sum_{i}^{N} x_{i}, \quad x_{i}$ : integer $>0$. Considering all compositions as equiprobable, we know (see [HL01]) that the number of parts $N$ is asymptotically Gaussian, $\nu \rightarrow \infty$ :

$$
N \sim \mathcal{N}\left(\frac{v}{2}, \frac{v}{4}\right),
$$

and that the part sizes are asymptotically id $\operatorname{GEOM}(1 / 2)$ and independent. Consider now $n$ random variables (R.V.), $\operatorname{GEOM}(1 / 2)$ and defi ne the indicator R.V. ${ }^{\dagger}$

$$
Y_{i}:=\text { घvalue } i \text { appears among these } n \text { R.V. } \rrbracket
$$

Then, asymptotically, $n \rightarrow \infty$, the $Y_{i}$ are independent. The fi rst empty part value, i.e the fi rst $k$ such that $Y_{k}=0$, is of order $O(\log n)$. Here and in the sequel, $\log :=\log _{2}, L:=\ln 2$. Similarly, the maximum part

\footnotetext{
$\dagger$ Here we use the indicator function notation proposed by Knuth et al. [GKP89].

1365-8050 @ 2003 Discrete Mathematics and Theoretical Computer Science (DMTCS), Nancy, France
} 
size is also of order $O(\log n)$, as well as the number $Y$ of distinct values (part sizes): $Y=\sum_{1}^{\infty} Y_{i}$. The asymptotic distributions and moments of these R.V. are also given in [HL01]. We know (see Hwang and Yeh [HY97]) that

$$
\mathbb{E}(Y) \sim \log n+\gamma / L-1 / 2+\beta(\log n)+O(1 / n)
$$

where $\beta$ is a small periodic function of $\log n$, and the distribution of $Y$ is highly concentrated around its mean, with a $O(1)$ range. All these distributions depend on $\log n$. Hence, with (1), the same R.V. related to $v$ are asymptotically equivalent by replacing $\log n$ by $\log v-1$ (see [HL01]).

In this article we consider a variant of the distinctness: the number $X(m)$ of distinct parts of multiplicity $m$ that we call the $m$-distinctness. A fi rst motivation is a question asked by Wilf for random compositions: what is the asymptotic value of the probability $P(m, v)$ that a randomly chosen part size in a random composition of an integer $v$ has multiplicity $m$. (The corresponding problem for random partitions has been analyzed in Corteel et al. [CPSW99]). Of course, here,

$$
P(m, v)=\mathbb{E}(X(m, v) / Y(v)),
$$

where we explicitly show the dependence on $v$. But, as already mentioned, $Y(v)$ has asymptotically the same distribution as $Y$ (with $\log n$ replaced by $\log v-1$ ). On the other side, $Y$ is highly concentrated around its mean . Hence, asymptotically, as shown in Hitczenko, Savage [HS99] and Hitczenko et al [HRS02], for $m=O(1)$,

$$
P(m, v) \sim \mathbb{E}(X(m, v)) / \mathbb{E}(Y(v)) .
$$

Here, we investigate, from a probabilistic point of view, the first full part, the maximum part size and the distribution of $X(m, v)$. We obtain asymptotically, as $v \rightarrow \infty$, the moments and an expression for a continuous distribution $\varphi$, the (discrete) distribution of $X(m, v)$ being computable from $\varphi$. We will see that, again, all asymptotic distributions for some multiplicity $m$ depend only on $\log n$. Hence, the same R.V. related to $v$ are again simply obtained by replacing $\log n$ by $\log v-1$. The paper is organized as follows: in Section 2, we consider a fi xed multiplicity $m=O(1)$. We analyze the moments, the fi rst full part, the maximum part size, and the distribution of $X(m)$. Section 3 is devoted to large multiplicity $m$. Section 4 concludes the paper. Due to length constraints, some proofs have been briefly presented.

In this section, we are interested in the properties of the R.V.:

$X_{i}(m):=\llbracket$ value $i$ appears among the $n \operatorname{GEOM}(1 / 2)$ R.V. with multiplicity $m$, for fi xed $m=O(1) \rrbracket$. Of course,

$$
\operatorname{Pr}\left[X_{i}(m)=1\right]=\left(\begin{array}{c}
n \\
m
\end{array}\right)\left(1 / 2^{i}\right)^{m}\left(1-1 / 2^{i}\right)^{n-m} .
$$

We immediately see that the dominant range is given by $i=\log n+O(1)$. To the left and the right of this range, $\operatorname{Pr}\left[X_{i}(m)=1\right] \sim 0$. Within the range, $\operatorname{Pr}\left[X_{i}(m)=1\right]$ is asymptotically equivalent to a Poisson distribution:

$$
\operatorname{Pr}\left[X_{i}(m)=1\right] \sim \frac{1}{m !}\left(n / 2^{i}\right)^{m} \exp \left(-n / 2^{i}\right),
$$

and, with $X(m):=\sum_{1}^{\infty} X_{i}(m)$,

$$
\mathbb{E}(X(m)) \sim G(n, m),
$$

where, using the "sum splitting technique" as described in Knuth [Knu73], p.131,

$$
G(n, m):=\frac{1}{m !} \sum_{i=1}^{\infty}\left(n / 2^{i}\right)^{m} \exp \left(-n / 2^{i}\right)
$$


which, for large $n$, can be analyzed using Mellin transforms: see Flajolet et al. [FGD95]. It is well known that the dominant value is given by some constant. The oscillatory part has a very small amplitude, usually of order $10^{-5}$. Indeed, set $f(y):=y^{m} e^{-y}$. We obtain

$$
G(n, m)=\frac{1}{m !} \sum_{i=1}^{\infty} f\left(n / 2^{i}\right)
$$

the Mellin transform of which is

$$
G^{*}(s)=\frac{\Gamma(m+s)}{m !} \frac{2^{s}}{1-2^{s}},
$$

defi ned in the fundamental strip $\langle-m, 0\rangle$. To the right of this strip, the poles of $G^{*}(s)$ are a simple pole at $s=0$, and simple poles at $s=\chi_{k}:=2 k \pi i / L(k \neq 0)$. The singular expansion of $G^{*}(s)$ is given by

$$
G^{*}(s) \asymp\left[\frac{\Gamma(m)}{L m ! s}\right]+\sum_{k \neq 0} \frac{\Gamma\left(m+\chi_{k}\right)}{L m !\left(s-\chi_{k}\right)} .
$$

This leads, by converse mapping, to

$$
G(n, m) \sim \frac{1}{m L}+\beta_{0}(\log n)+O(1 / n)
$$

where $\beta_{0}$ is a small periodic function of $\log n$ :

$$
\beta_{0}\left(\log _{2} n\right):=\sum_{k \neq 0} \frac{\Gamma\left(m+\chi_{k}\right)}{L m !} n^{-\chi_{k}}=\sum_{k \neq 0} \frac{\Gamma\left(m+\chi_{k}\right)}{L m !} e^{-2 \pi i k \log n} .
$$

In the sequel, $\beta .(\log n)$ will always denote (small) periodic functions. As $n \sim \mathcal{N}\left(\frac{v}{2}, \frac{v}{4}\right)$, we just have to replace $\log n$ by $\log v-1$. So we recover the mean already computed in Hitczenko and Savage, [HS99] and Hitczenko, Rousseau and Savage, [HRS02]. To compute all moments, we must check that the $X_{i}$ are asymptotically independent. We could proceed as was done in [HL01] for the $Y_{i}$, but we follow here another route. Let us consider $\Pi_{n}=\mathbb{E}\left(z^{X}\right)$. We obtain

Theorem 1.1.

$$
\Pi_{n} \sim \prod_{l=1}^{\infty}\left[\left(1-\frac{1}{m !}\left(n / 2^{l}\right)^{m} e^{-n / 2^{l}}\right)+z \frac{1}{m !}\left(n / 2^{l}\right)^{m} e^{-n / 2^{l}}\right], n \rightarrow \infty
$$

Proof. We use an urn model, as in Sevastyanov and Chistyakov, [SČ64] and Chistyakov, [Chi67], and the Poissonization method (see, for instance Jacquet and Szpankowski [JS98] for a general survey). If we Poissonize, with parameter $\tau$, the number of balls (i.e the number $n$ of R.V. here), the generating function of $X_{l}$ is given from (2), by

$$
\left(1-\frac{1}{m !}\left(\tau / 2^{l}\right)^{m} e^{-\tau / 2^{l}}\right)+z \frac{1}{m !}\left(\tau / 2^{l}\right)^{m} e^{-\tau / 2^{l}}
$$

₹ The symbol $\asymp$ is used to denote the fact that two functions are of the same asymptotic order. 
and we have independency of cells occupation. This leads to

$$
e^{-\tau} \sum_{n} \frac{\tau^{n}}{n !} \Pi_{n}=\prod_{l=1}^{\infty}\left[\left(1-\frac{1}{m !}\left(\tau / 2^{l}\right)^{m} e^{-\tau / 2^{l}}\right)+z \frac{1}{m !}\left(\tau / 2^{l}\right)^{m} e^{-\tau / 2^{l}}\right] .
$$

Hence, by Cauchy, we obtain $\Pi_{n}=\frac{n !}{2 \pi i} \int_{\Gamma} \exp \{n f(\tau)\} d \tau / \tau$, where $\Gamma$ is inside the analyticity domain of the integrand and encircles the origin, and

$$
f(\tau):=-\log \tau+\tau / n+\frac{1}{n} \sum_{l=1}^{\infty} \ln \left[\left(1-\frac{1}{m !}\left(\tau / 2^{l}\right)^{m} e^{-\tau / 2^{l}}\right)+z \frac{1}{m !}\left(\tau / 2^{l}\right)^{m} e^{-\tau / 2^{l}}\right] .
$$

By standard saddle-point method (see, for instance, Flajolet and Sedgewick, [FS94]), we look for $\tau^{*}$ such that $f^{\prime}\left(\tau^{*}\right)=0$, with

$$
f^{\prime}(\tau)=-1 / \tau+1 / n-\frac{z-1}{n \tau} \sum_{l=1}^{\infty} \frac{\left(\tau / 2^{l}\right)^{m+1}-m\left(\tau / 2^{l}\right)^{m}}{m ! \exp \left(\tau / 2^{l}\right)-\left(\tau / 2^{l}\right)^{m}+z\left(\tau / 2^{l}\right)^{m}}
$$

But, again by Mellin, for fi xed $z>0$,

$$
\sum_{l=1}^{\infty} \frac{\left(\tau / 2^{l}\right)^{m+1}-m\left(\tau / 2^{l}\right)^{m}}{m ! \exp \left(\tau / 2^{l}\right)-\left(\tau / 2^{l}\right)^{m}+z\left(\tau / 2^{l}\right)^{m}} \sim C+\beta \cdot(\log \tau)
$$

with

$$
C:=\int_{0}^{\infty} \frac{y^{m+1}-m y^{m}}{m ! \exp (y)-y^{m}+z y^{m}} d y / L
$$

Hence $\tau^{*} \sim n+C$. It is easily checked that $C=0$. Finally, $\Pi_{n} \sim \frac{n ! e^{n f\left(\tau^{*}\right)}}{\sqrt{2 \pi} \tau^{*} \sqrt{n f^{\prime \prime}\left(\tau^{*}\right)}}$, and, by Stirling, we easily derive the theorem.

Theorem 1.1 confi rms the asymptotic independence assumption.

\subsection{The moments of $X(\mathrm{~m})$}

We now have all necessary ingredients to compute the moments. The variance of $X(m)$ is now easily derived: we obtain, by Mellin,

$$
\begin{aligned}
\operatorname{VAR}(X(m)) & \sim \frac{1}{m !} \sum_{1}^{\infty}\left(n / 2^{i}\right)^{m} \exp \left(-n / 2^{i}\right)\left[1-\frac{1}{m !}\left(n / 2^{i}\right)^{m} \exp \left(-n / 2^{i}\right)\right] \\
& \sim \int_{0}^{\infty} e^{-y} \frac{y^{m}}{m !}\left(1-e^{-y} \frac{y^{m}}{m !}\right) \frac{d y}{L y}+\beta_{1}\left(\log _{2} n\right) \\
& =\frac{1}{m L}-\frac{(2 m-1) !}{L m !^{2} 2^{2 m}}+\beta_{1}\left(\log _{2} n\right) .
\end{aligned}
$$


The other moments can be derived as follows. We obtain, setting $z=e^{s}$,

$$
\begin{aligned}
\ln \left(\Pi_{n}\right) \sim S_{2} & =\sum_{l=1}^{\infty} \ln \left[1+\left(e^{s}-1\right) \frac{1}{m !}\left(n / 2^{l}\right)^{m} \exp \left(-n / 2^{l}\right)\right] \\
& =\sum_{i=1}^{\infty} \frac{(-1)^{i+1}\left(e^{s}-1\right)^{i} V_{i}}{i}, \text { with } \\
V_{i} & :=\sum_{l=1}^{\infty}\left[\frac{1}{m !}\left(n / 2^{l}\right)^{m}\right]^{i} \exp \left(-i n / 2^{l}\right) .
\end{aligned}
$$

The centered moments of $X(m)$ can be obtained by analyzing

$$
S_{3}:=\exp \left[S_{2}-s V_{1}\right]
$$

Again, by Mellin, we obtain

$$
V_{i} \sim B_{i}+\beta_{i}(\log n)
$$

with

$$
B_{i}=\int_{0}^{\infty}\left[\frac{y^{m}}{m !}\right]^{i} e^{-i y} \frac{d y}{L y}=\frac{(i m-1) !}{m !^{i} L i^{i m}},
$$

and fi nally, the centered moments are given by

$$
\begin{aligned}
\tilde{\sigma}^{2}:=\operatorname{VAR}(X(m)) & \sim \frac{1}{m L}-\frac{(2 m) !}{2 L m !^{2} 2^{2 m} m} \\
\tilde{\mu}_{3}:=\mu_{3}(X(m)) & \sim \frac{1}{m L}-\frac{3(2 m) !}{2 L m !^{2} 2^{2 m} m}+\frac{2(3 m) !}{3 L m !^{3} 3^{3 m} m} \\
\tilde{\mu}_{4}:=\mu_{4}(X(m)) & \sim \frac{1}{m L}+\frac{3}{m^{2} L^{2}}-\frac{3(4 m) !}{2 L m !^{4} 4^{4 m} m}+\frac{4(3 m) !}{L m !^{3} 3^{3 m} m} \\
& -\frac{7(2 m) !}{2 L m !^{2} 2^{2 m} m}-\frac{3(2 m) !}{3 L^{2} m !^{2} 2^{2 m} m^{2}}+\frac{3(2 m) !^{2}}{4 L^{2} m !^{4} 2^{4 m} m^{2}} .
\end{aligned}
$$

The neglected terms are made of periodic functions $\beta .(\log n)$ and of $O\left(\frac{1}{n}\right)$ contributions.

Again, the centered moments (of order $\geq 2$ ) of $X$ related to a composition of $v$ are given by the same expressions.

For $n=20000, m=2$, we have done a simulation (of $T=4000$ sets). We obtain the results of Table 1 (the probability related moments are explained later on). For an easy comparison, we give here only four signifi cant digits.

\subsection{The maximum part size of multiplicity $m$}

The maximum part size $\mathcal{M}_{n}(m)$ of multiplicity $m$ is such that

$$
\operatorname{Pr}\left(\mathcal{M}_{n}(m)<k\right) \sim \prod_{i=k}^{\infty}\left[1-\frac{1}{m !}\left(n / 2^{i}\right)^{m} \exp \left(-n / 2^{i}\right)\right] .
$$




\begin{tabular}{|l|l|l|l|}
\hline & $\begin{array}{l}\text { Theoretical } \\
\text { asymptotic value }\end{array}$ & Observed value & $\begin{array}{l}\text { Probability } \\
\text { related value }\end{array}$ \\
\hline mean & $.7213 \ldots$ & $.7345 \ldots$ & $.7214 \ldots$ \\
variance $\ldots$ & $.5861 \ldots$ & $.5945 \ldots$ & $.5863 \ldots$ \\
$\mu_{3}$ & $.3750 \ldots$ & $.3752 \ldots$ & $.3752 \ldots$ \\
$\mu_{4}$ & $1.1197 \ldots$ & $1.1341 \ldots$ & $1.1198 \ldots$ \\
\hline
\end{tabular}

Tab. 1: Moments, $n=20000, m=2$.

Set $\eta:=L k-\ln n$. This leads, with $\eta=O(1)$, to

$$
\operatorname{Pr}\left(\mathcal{M}_{n}(m)<k\right) \sim \varphi_{1}(m, \eta)
$$

with

$$
\varphi_{1}(m, \eta)=\prod_{j=0}^{\infty}\left[1-\frac{1}{m !} e^{-m(\eta+L j)} e^{-e^{-(\eta+L j)}}\right] .
$$

Figure 1 gives $\varphi_{1}(m, \eta)$ for $m=1, \ldots, 4$, bottom to top. It appears that for $\eta \rightarrow-\infty, \varphi_{1}(m, \eta)$ seems to converge to some value, which of course corresponds to

$$
P(m, 0):=\operatorname{Pr}(X(m)=0),
$$

but a closer view reveals the usual fluctuations, shown in Figure 2, for $m=2$. Set $\psi(n):=\log n-$ $\lfloor\log n\rfloor$ (fractional part). With $\eta=L(-6-\psi(20000)$ ), we obtain $P(2,0)=.4489079864 \ldots$, which will be compared later on with a direct expression.

Similarly, we derive

$$
\operatorname{Pr}\left(\mathcal{M}_{n}(m)=k-1\right) \sim \varphi_{2}(m, \eta)=\varphi_{1}(m, \eta) e^{-m(\eta-L)} e^{-e^{-(\eta-L)}} / m ! .
$$

Figure 3 gives $\varphi_{2}(m, \eta)$ for $m=1, \ldots, 4$, (more and more concentrated as $m$ increases).

Our simulation for $n=20000, m=2$ of $T=4000$ sets leads to Figure $4\left(\varphi_{1}\right.$, observed $=$ circle, asymptotic $=$ line $)$ and Figure $5\left(\varphi_{2}\right.$, observed $=$ circle, asymptotic $=$ line $)$. Again, for compositions, we replace $\log n$ by $\log v-1$.

\subsection{First full part value of multiplicity $m$}

Another variable of interest is the fi rst $k$ such that $X_{k}=1$, i.e we are interested in the probability

$$
\operatorname{Pr}\left[X_{i}=0, i=1 \cdots k-1, X_{k}=1\right] .
$$

Note that this is the opposite situation of the $Y_{k}$ case (see [HL01]), where we looked for the first $k$ such that $Y_{k}=0$. The probability is asymptotically given by

$$
\prod_{i=1}^{k-1}\left[1-\frac{1}{m !}\left(n / 2^{i}\right)^{m} \exp \left(-n / 2^{i}\right)\right] \frac{1}{m !}\left(n / 2^{k}\right)^{m} \exp \left(-n / 2^{k}\right) .
$$


Again, we set $\eta:=L k-\ln n$. This leads asymptotically, with $\eta=O(1)$ to

$$
\operatorname{Pr}\left[X_{i}=0, i=1 \cdots k-1, X_{k}=1\right] \sim \varphi_{3}(m, \eta),
$$

with

$$
\begin{aligned}
& \varphi_{3}(m, \eta)=\varphi_{4}(m, \eta) \frac{1}{m !} e^{-m \eta} e^{-e^{-\eta}}, \\
& \varphi_{4}(m, \eta)=\prod_{j=1}^{\infty}\left[1-\frac{1}{m !} e^{-m(\eta-L j)} e^{-e^{-(\eta-L j)}}\right] .
\end{aligned}
$$

Again, for compositions, we replace $\log n$ by $\log v-1$. Figure 6 gives $\varphi_{4}(2, \eta)$ and Figure 7 gives $\varphi_{4}(2, \eta)$ for large values of $\eta$. Again, this is oscillating and corresponds to $P(2,0)$.

\subsection{Asymptotic distribution of $X(\mathrm{~m})$}

The analysis is rather similar to the one we used in [Lou87] and [HL01]. First of all we have, for any fi xed $k=O(\log n)$,

$$
P(m, 0) \sim \varphi_{4}(\eta) \varphi_{1}(\eta)
$$

Let us choose $k=\lfloor\log n\rfloor$. This leads to $\eta=-L \psi(n)$ and we obtain a periodic function of $\psi$ :

$$
P(m, 0) \sim \varphi_{4}[-L \psi(n)] \varphi_{1}[-L \psi(n)]
$$

shown in Figure 10 for $m=2$. For $n=20000, m=2$, the numerical value of $P(2,0)$ is exactly the same as before. Now we turn to $P(m, j):=\operatorname{Pr}(X(m)=j)$. We take advantage of the fact that all urns are empty before the fi rst occupied urn, $k-1$ say. Then, again with $\eta:=L k-\ln n$,

$$
\begin{aligned}
P(m, 1) & \sim \sum_{k} \varphi_{3}(\eta-L) \varphi_{1}(\eta) \\
P(m, 2) & \sim \sum_{k} \varphi_{3}(\eta-L) \varphi_{1}(\eta) \sum_{r_{1} \geq k}\left\{\frac{1}{m !}\left(n / 2^{r_{1}}\right)^{m} \exp \left(-n / 2^{r_{1}}\right) /\left[1-\frac{1}{m !}\left(n / 2^{r_{1}}\right)^{m} \exp \left(-n / 2^{r_{1}}\right)\right]\right\}
\end{aligned}
$$

and more generally,

$$
\begin{aligned}
& P(m, u+1) \sim \sum_{k} \varphi_{3}(\eta-L) \varphi_{1}(\eta) . \\
& \quad \cdot \sum \llbracket r_{1}>r_{2}>. .>r_{u}, r_{j} \geq k \rrbracket \prod_{i=1}^{u}\left\{\frac{1}{m !}\left(n / 2^{r_{i}}\right)^{m} \exp \left(-n / 2^{r_{i}}\right) /\left[1-\frac{1}{m !}\left(n / 2^{r_{i}}\right)^{m} \exp \left(-n / 2^{r_{i}}\right)\right]\right\}
\end{aligned}
$$

Now we set $r_{i}=k+w_{i}, l=k-\lfloor\log n\rfloor$ and we fi nally derive the following theorem

Theorem 1.2. Set $\psi(n):=\log n-\lfloor\log n\rfloor$, then

$$
P(m, u+1) \sim \sum_{l=-\infty}^{\infty} \varphi_{5}[L(l-\psi(n))],
$$


with

$\varphi_{5}(\eta)=\varphi_{3}(\eta-L) \varphi_{1}(\eta)$

$$
\cdot \sum \llbracket w 1>w 2>\ldots>w_{u}, w_{j} \geq 0 \rrbracket \prod_{i=1}^{u}\left\{\frac{1}{m !} e^{-m\left(\eta+L w_{i}\right)} e^{-e^{-\left(\eta+L w_{i}\right)}} /\left[1-\frac{1}{m !} e^{-m\left(\eta+L w_{i}\right)} e^{-e^{-\left(\eta+L w_{i}\right)}}\right]\right\}
$$

Note that, for compositions, we obtain asymptotically $\psi(n)=\psi(v)$. We get again periodic function of $\psi(n)$. We give in Figure 11 and Figure 12 the sums

$\sum_{i=0}^{3} P(2, i), \sum_{i=0}^{4} P(2, i)$. The effect of computing $P(2, i)$ with bounded indices (we limit the values of $w_{u}$ to 16$)$ becomes apparent at the $10^{-7}$ precision.

Figure 13 gives $P(m, i), m=1, \ldots, 4$, (from top to bottom to the right of $i=2$ ). The distributions become more concentrated as $m$ increases.

Finally, we compare the observed distribution of $X(2)$ with the asymptotic one in Figure 14 (observed = circle, asymptotic $=$ line). Apart from $i=0$ the fi t is quite good. The "Probability related values" moments given in Table 1 are computed with the distribution $P(2, i)$.

\section{Large multiplicity $m$}

\subsection{Fixed number of parts $n$}

It is now clear that large $m$ are related to small integer values $i$. More precisely, the number $M_{i}$ of integers equal to $i$ is asymptotically given by a Gaussian:

$$
\operatorname{Pr}\left(M_{i}=m\right) \sim \exp \left\{-\left(m-n / 2^{i}\right)^{2} /\left[2 n / 2^{i}\left(1-1 / 2^{i}\right)\right]\right\} / \sqrt{2 \pi n / 2^{i}\left(1-1 / 2^{i}\right)} .
$$

The means $n / 2^{i}, i=1,2, \ldots$ are given by $n / 2, n / 4, \ldots$, separated by $n / 4, n / 8, \ldots$ which shows that the Gaussians (4) are asymptotically exponentially distinct in the sense that some common intervals, for instance $m \in\left[3 n / 2^{i+2}-n / 2^{i+3} . .3 n / 2^{i+2}+n / 2^{i+3}\right]$ have asymptotically small probability measures. So for any large value $m$, only one value

$$
i=\operatorname{round}[\log (n / m)]
$$

is related to $m$ and $X(m)$ has only two possible values: $\{0,1\}$. The following events are equivalent: $\llbracket X_{i}(m)=1 \rrbracket \equiv \llbracket M_{i}=m \rrbracket$. The probability (4) is small, of order at most $O(1 / \sqrt{m})$. Figure 15 gives $\operatorname{Pr}\left(X_{i}(m)=1\right)$ for $n=2000$ (fi rst three ranges, $\left.i=1,2,3\right)$ and Figure 16 gives the corresponding distribution functions, together with the observed values provided by a simulation of $T=2000$ sets (observed $=$ circle, asymptotic $=$ line).

An interesting check would be to recover the dominant term of the mean of $Y: \mathbb{E}(Y) \sim \log n$. Choose $\tilde{j}:=\alpha \log n, 0<\alpha<1$ which corresponds, by (5), to $\tilde{m}=n^{1-\alpha}$. For each $i \leq \tilde{j}$, by Euler-McLaurin,

$$
\sum_{m=\left\lfloor 3 n / 2^{i+2}\right\rfloor}^{\left\lfloor 3 n / 2^{i+1}\right\rfloor} \exp \left\{-\left(m-n / 2^{i}\right)^{2} /\left[2 n / 2^{i}\left(1-1 / 2^{i}\right)\right]\right\} / \sqrt{2 \pi n / 2^{i}\left(1-1 / 2^{i}\right)} \sim 1,
$$

and this contributes to $\mathbb{E}(Y)$ by $S_{1}=\tilde{j}$. On the other side, each $m<\tilde{m}$ contributes, by (3), with $\frac{1}{m L}$, with a total contribution

$$
S_{2}=\frac{1}{L} \sum_{1}^{\tilde{m}} 1 / m \sim \frac{1}{L} \ln \tilde{m} .
$$


The quantity $S_{1}+S_{2} \sim \log n$ as expected.

\subsection{Composition of $v$.}

Now the number of parts $N$ is such that (see(1))

$$
N \sim \mathcal{N}\left(\frac{v}{2}, \frac{v}{4}\right) .
$$

We obtain

$$
\mathbb{E}\left(M_{k}\right)=\frac{v}{2} \frac{1}{2^{k}}
$$

The asymptotic distribution of $M_{k}$ is obtained as follows. We derive, setting $\tilde{M}_{k}:=\left(M_{k}-n / 2^{k}\right) / \sqrt{v}$,

$$
\begin{aligned}
\mathbb{E} & {\left[\exp \left[i M_{k} \theta / \sqrt{v}\right]\right]=\mathbb{E}\left[\exp \left[i n \theta /\left(\sqrt{v} 2^{k}\right)+i \tilde{M}_{k} \theta\right]\right] } \\
& \sim \mathbb{E}\left[\exp \left[i n \theta /\left(\sqrt{v} 2^{k}\right)-\theta^{2} n /\left(2 v 2^{k}\right)\left(1-1 / 2^{k}\right)\right]\right] \\
& \sim \exp \left[i v \theta /\left(2 \sqrt{v} 2^{k}\right)-v \theta^{2} /\left(2 v 2.2^{k}\right)\left(1-1 / 2^{k}\right)+v / 8\left[i \theta /\left(\sqrt{v} 2^{k}\right)-\theta^{2} /\left(2 v 2^{k}\right)\left(1-1 / 2^{k}\right)\right]^{2}\right] \\
& \sim \exp \left[i \theta \sqrt{v} /\left(2.2^{k}\right)-\theta^{2} / 2\left[1 /\left(4.4^{k}\right)+1 /\left(2.2^{k}\right)\left(1-1 / 2^{k}\right)\right]\right], v \rightarrow \infty .
\end{aligned}
$$

The fi rst term confi rms (6). The second term shows that

$$
M_{k} \sim \mathcal{N}\left(\frac{v}{2} \frac{1}{2^{k}}, v \sigma_{m}^{2}\right)
$$

with

$$
\sigma_{m}^{2}=1 /\left(4.4^{k}\right)+1 /\left(2.2^{k}\right)\left(1-1 / 2^{k}\right)
$$

The conclusions of Sec. 2.2 are still valid.

\section{Conclusion}

Using various techniques from analysis and probability theory, we have analyzed the stochastic properties of the $m$-distinctness of random compositions. An interesting open problem would be be to extend our results to the Carlitz compositions, where two successive parts are different (see [LP02]).

\section{Acknowledgements}

The pertinent comments of the referees led to substantial improvements in the presentation. 


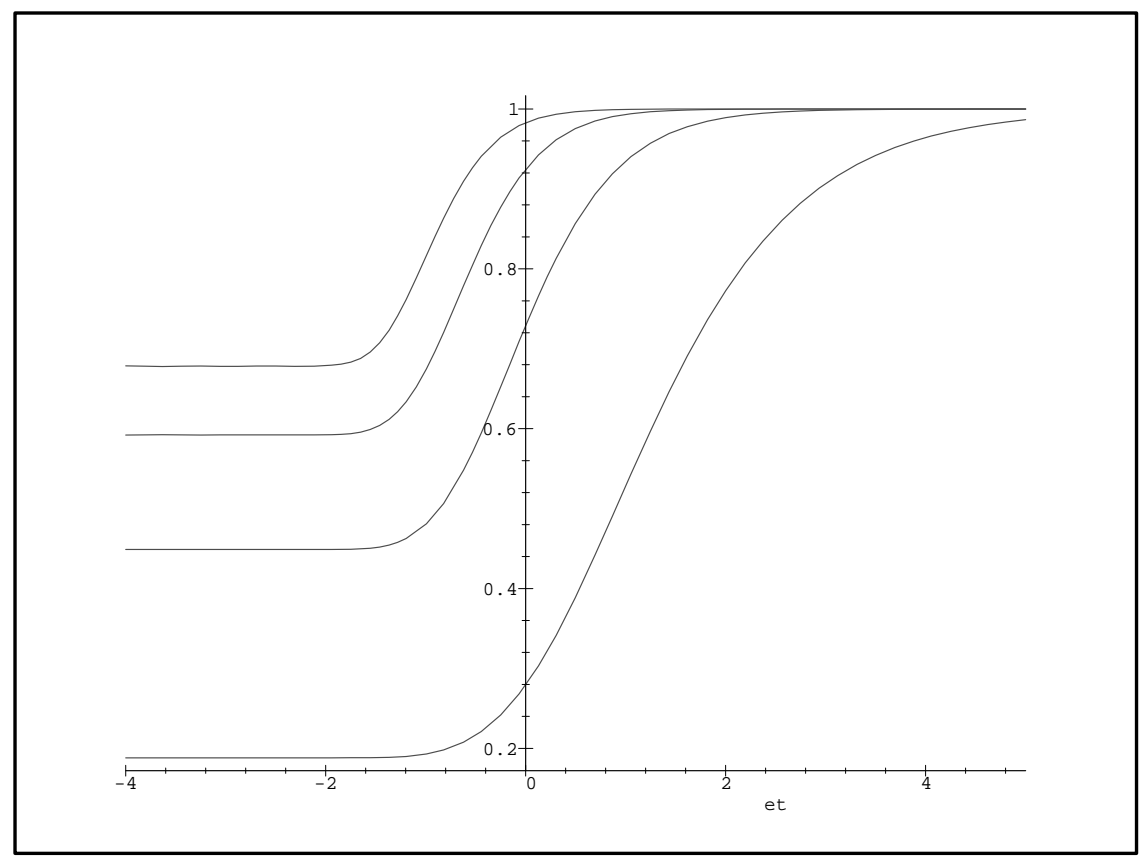

Fig. 1: $\varphi_{1}(m, \eta)$ for $m=1, \ldots, 4$, bottom to top

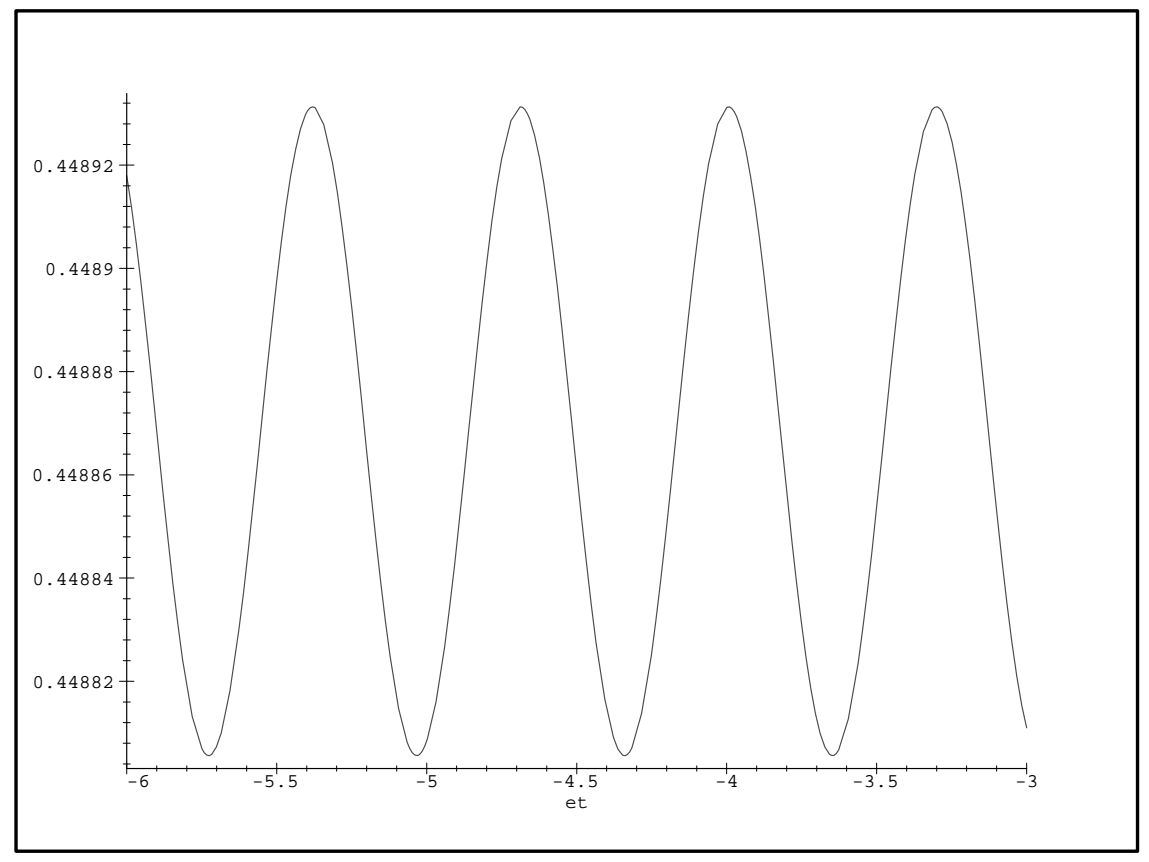

Fig. 2: $\varphi_{1}(2, \eta)$ for large negative values of $\eta$ 


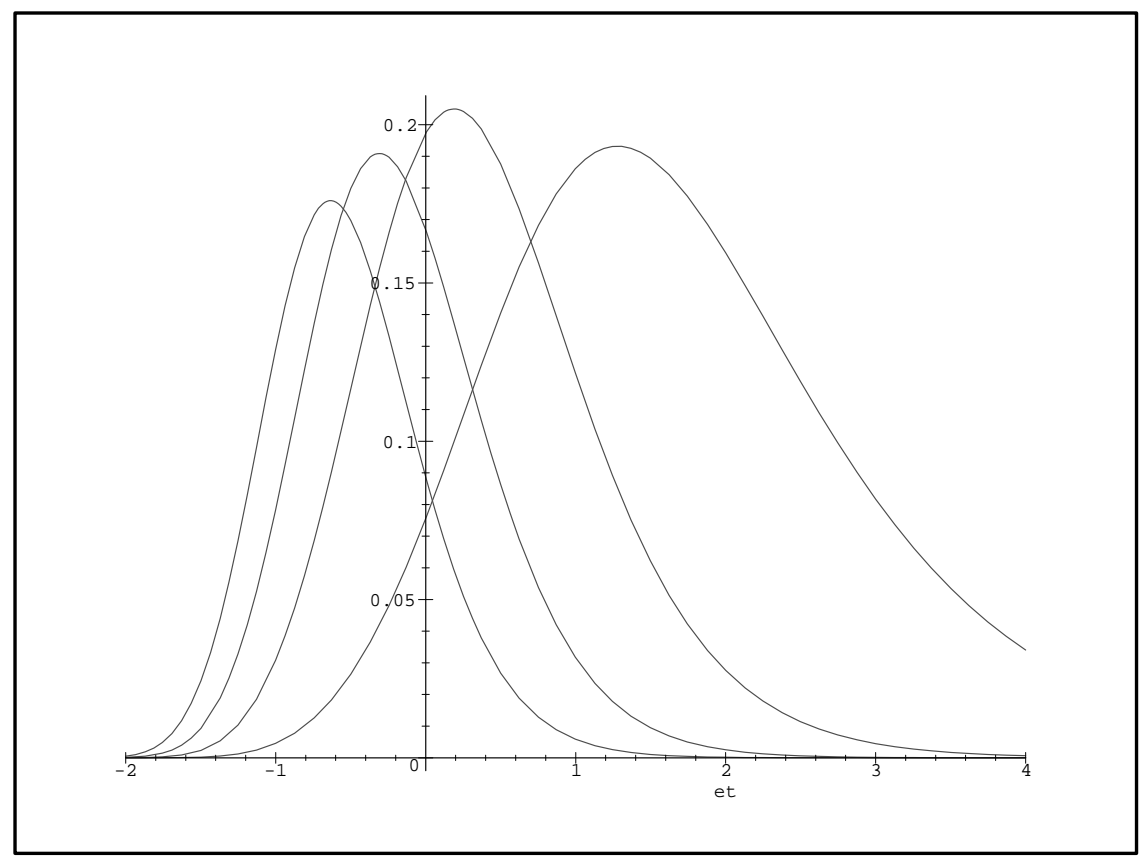

Fig. 3: $\varphi_{2}(m, \eta)$ for $m=1, \ldots, 4$

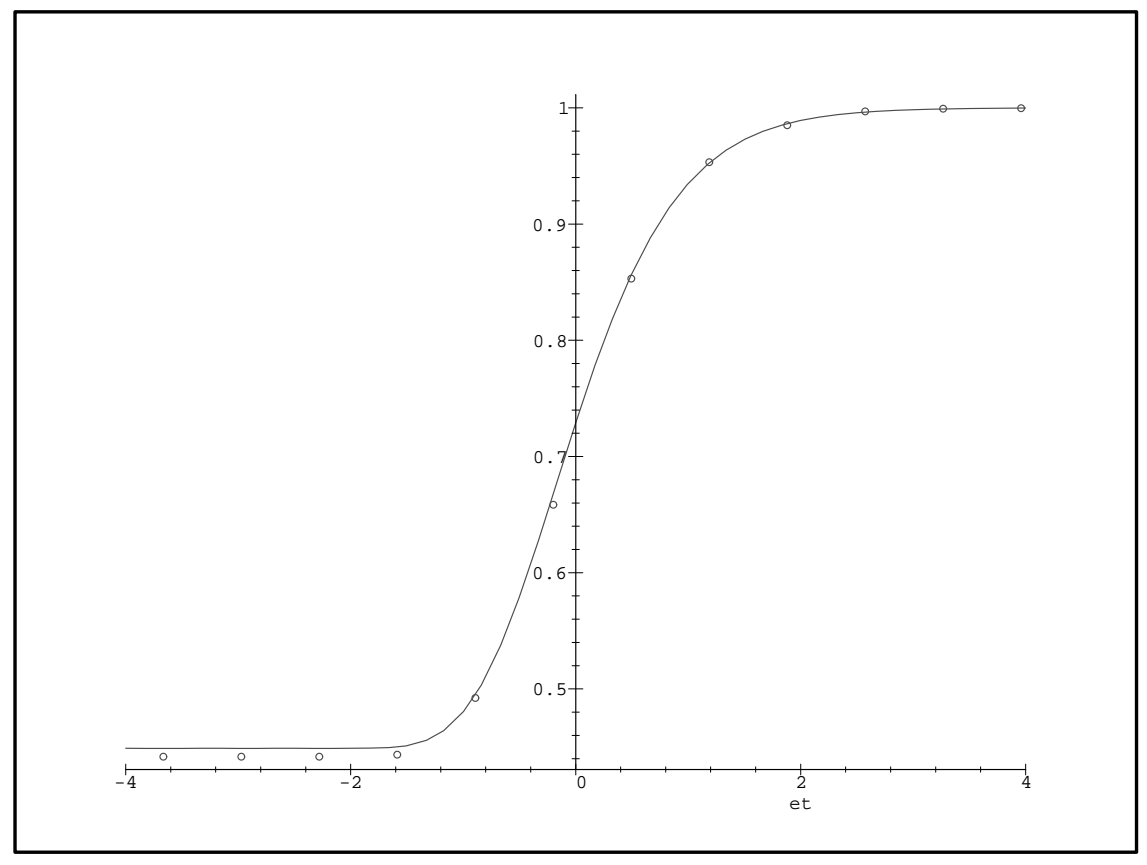

Fig. 4: Maximum part size distribution function $(m=2$, observed $=$ circle, asymptotic $=$ line $)$ 


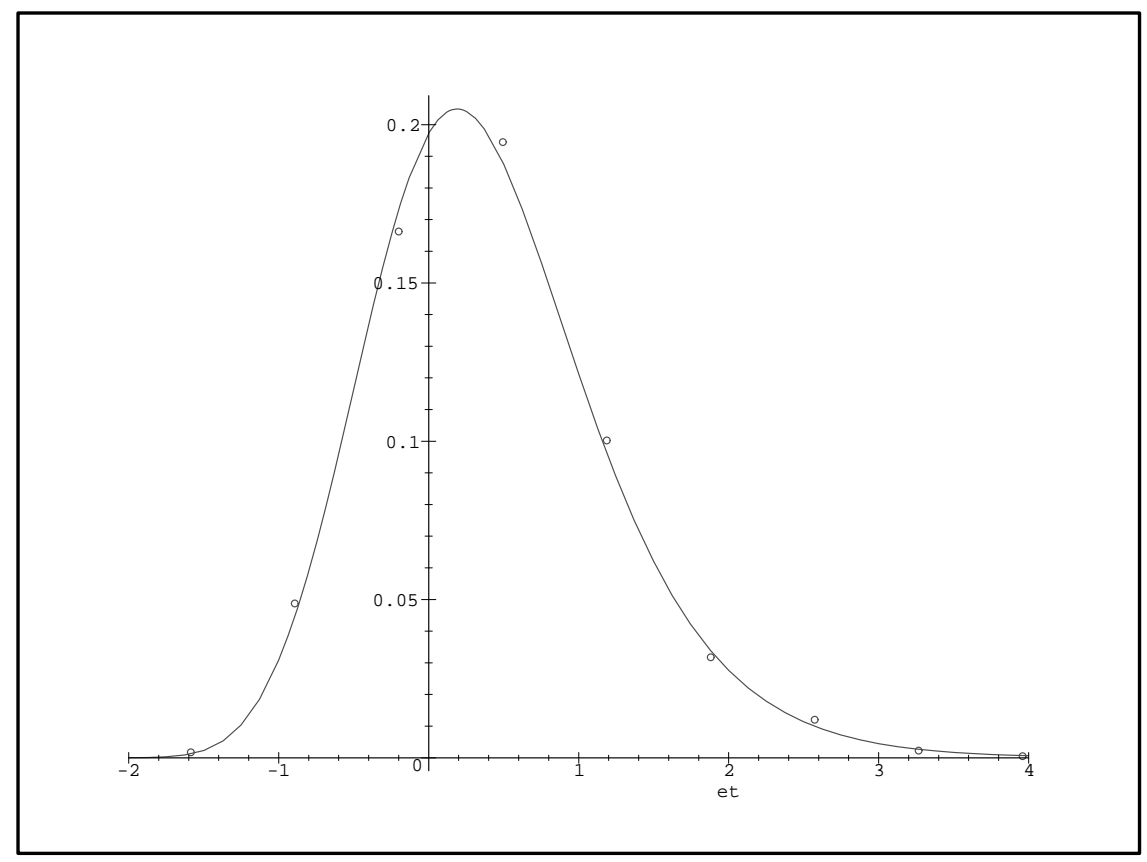

Fig. 5: Maximum part size distribution $(m=2$, observed $=$ circle, asymptotic $=$ line $)$

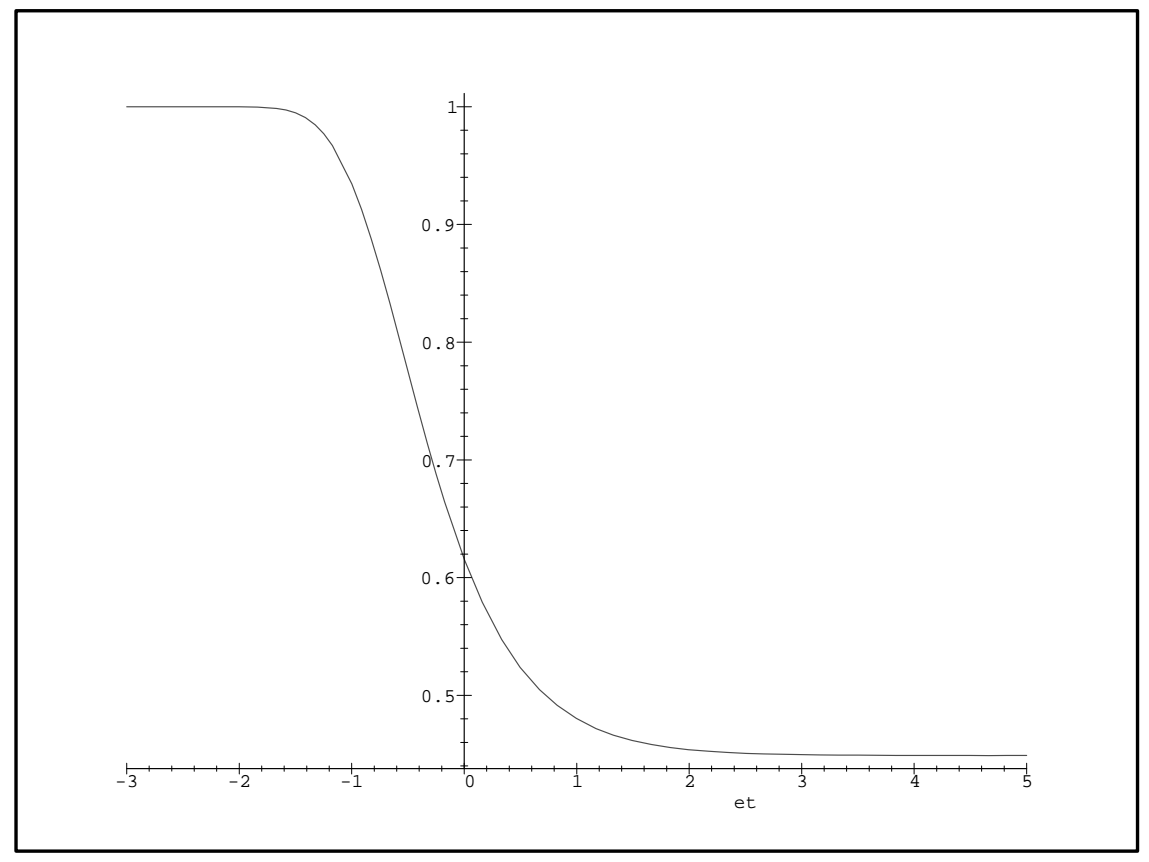

Fig. 6: $\varphi_{4}(2, \eta)$ 


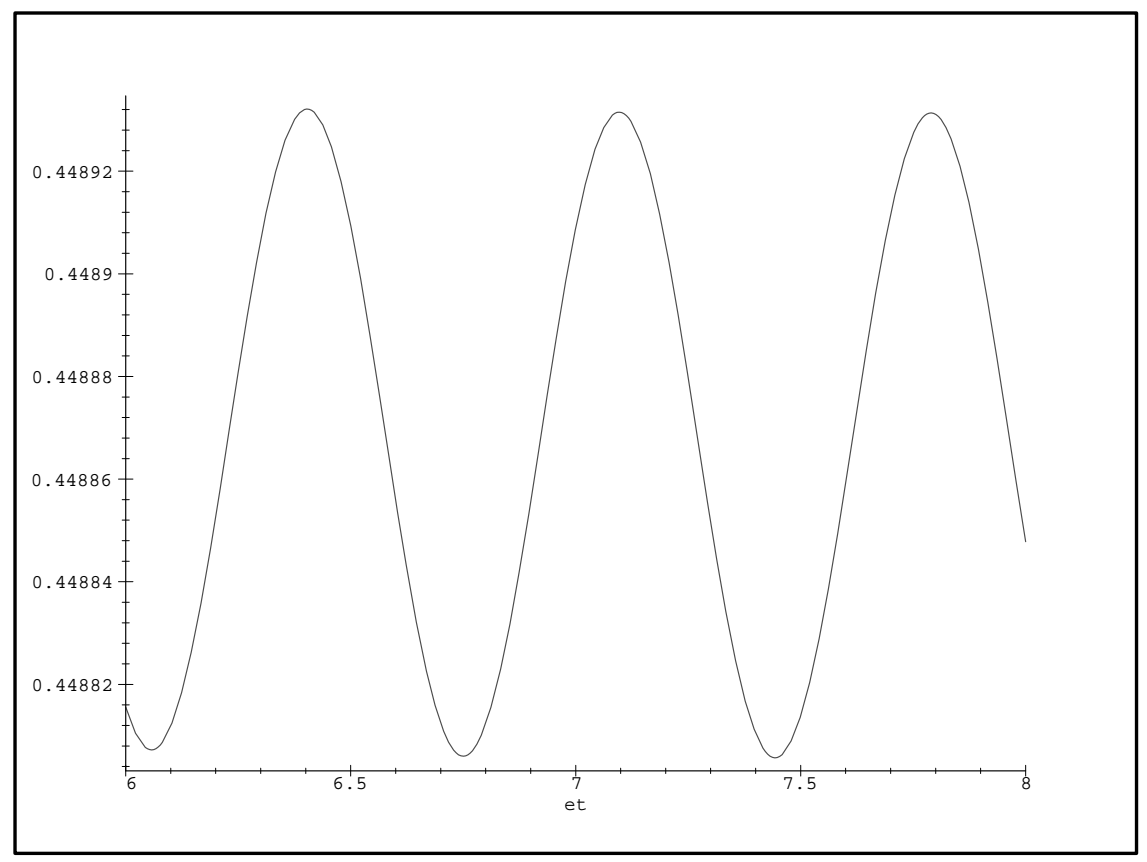

Fig. 7: $\varphi_{4}(2, \eta)$ for large values of $\eta$

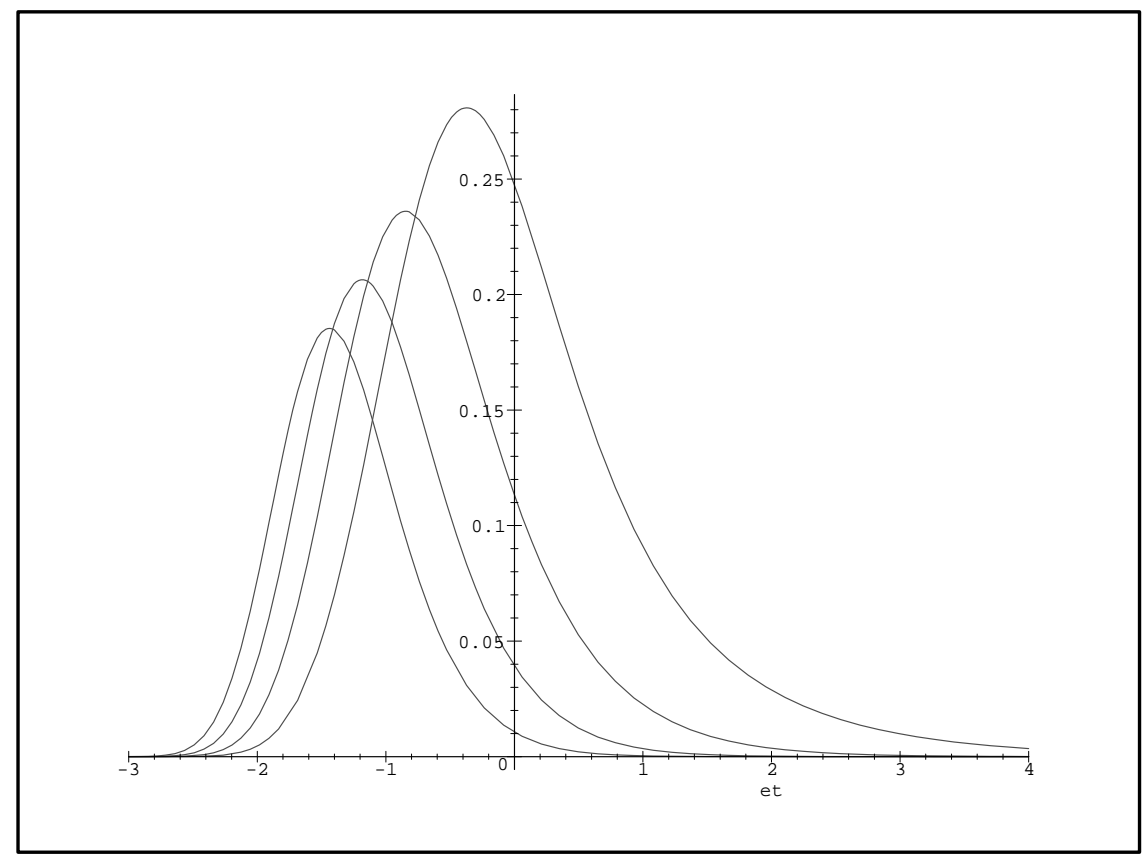

Fig. 8: $\varphi_{3}(2, \eta)$ for $m=1, \ldots, 4$. 


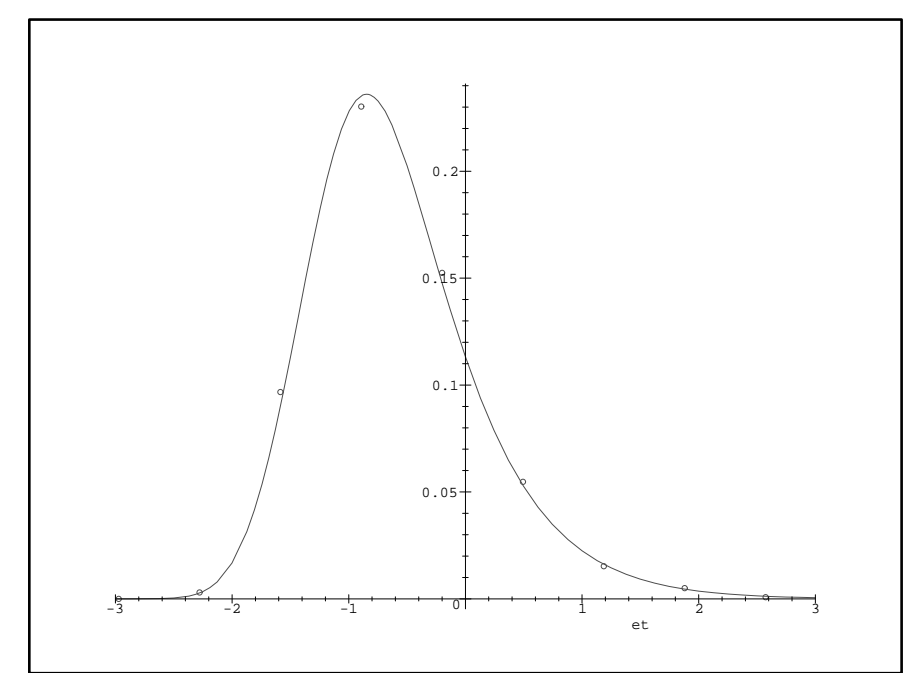

Fig. 9: First full part distribution $(m=2$, observed $=$ circle, asymptotic = line)

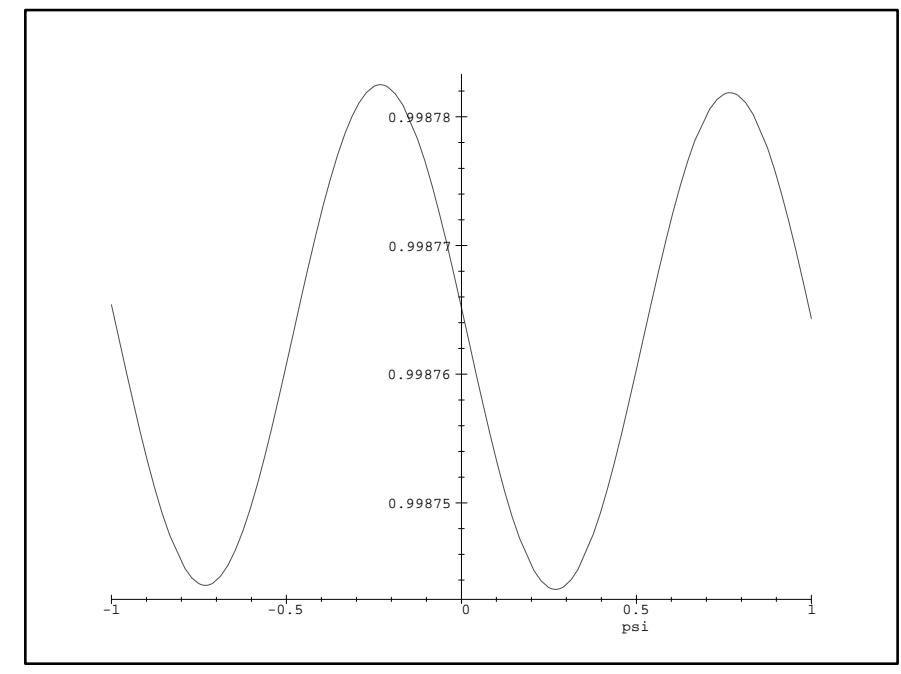

Fig. 11: $\sum_{i=0}^{3} P(2, i)$

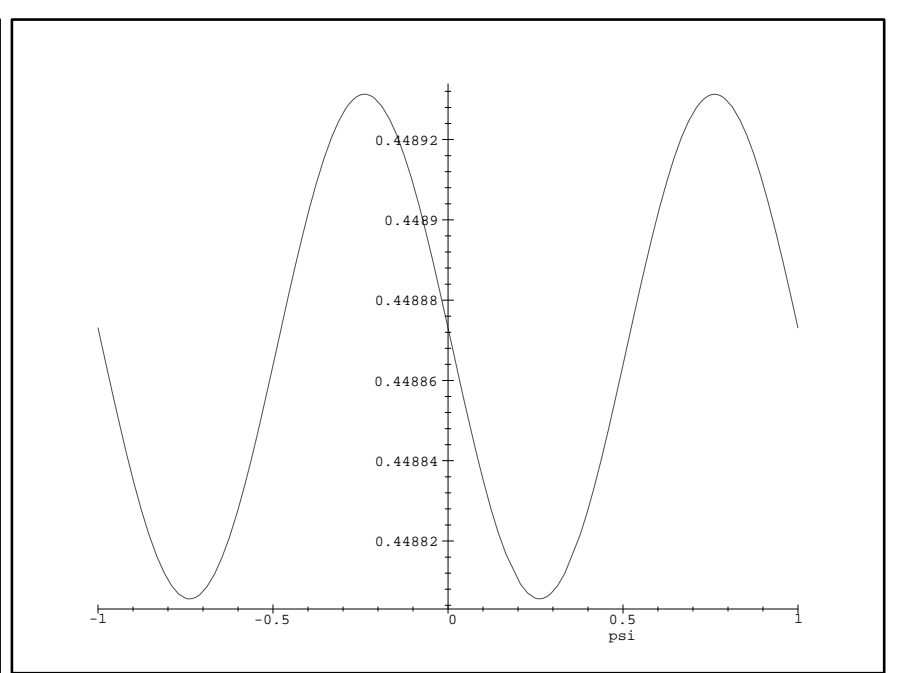

Fig. 10: $P(2,0)$ as a function of $\psi$

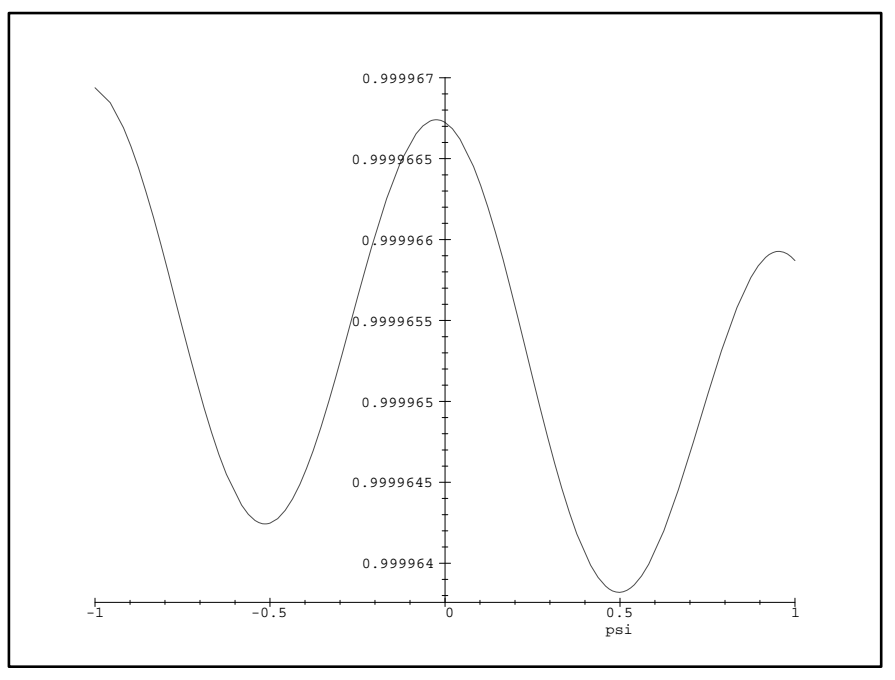

है

Fig. 12: $\sum_{i=0}^{4} P(2, i)$ 

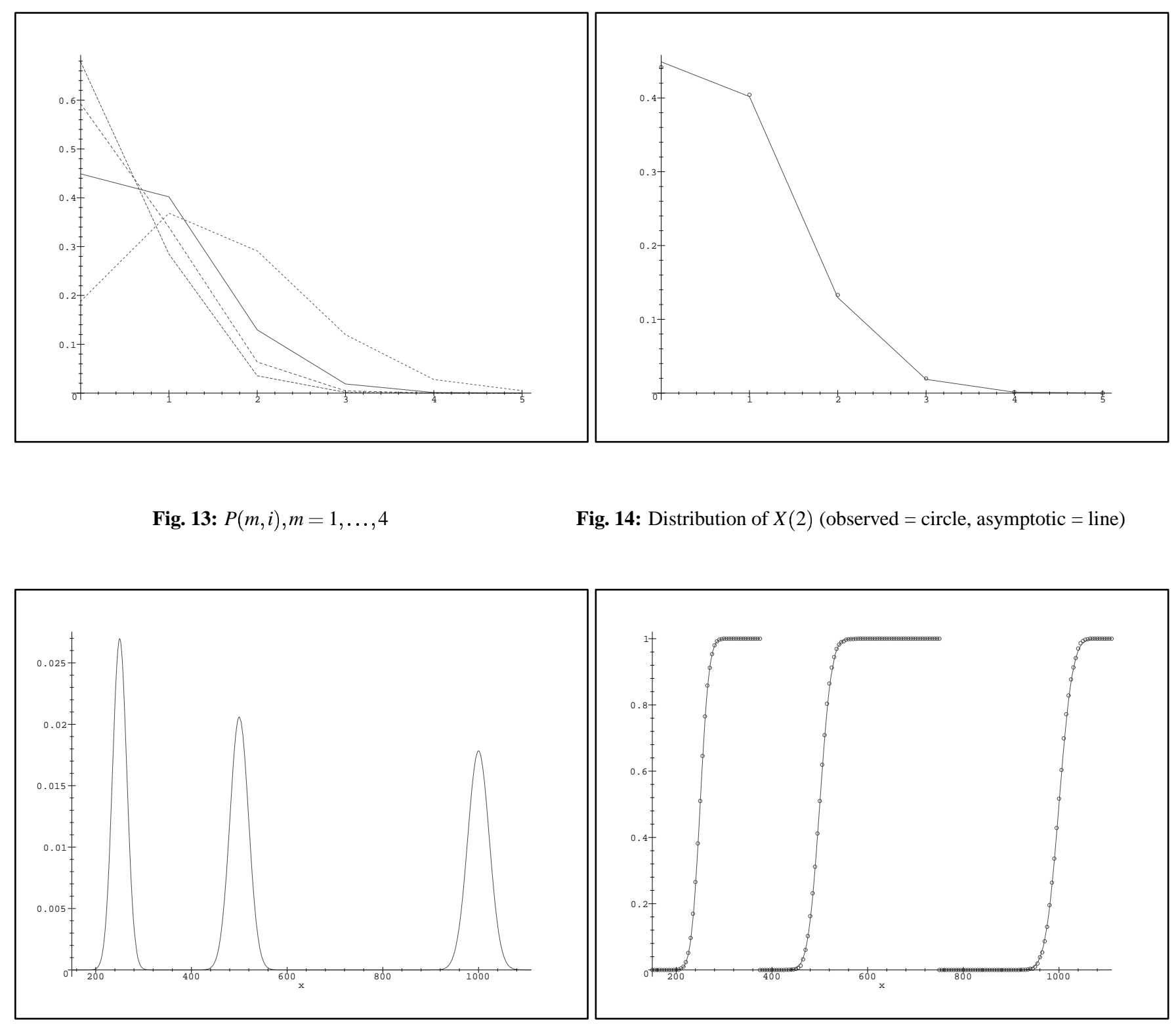

Fig. 15: $\operatorname{Pr}\left(X_{i}(m)=1\right), i=1, \ldots, 3$

Fig. 16: Distribution function of $M_{i}, i=1, \ldots, 3$ (observed $=$ circle, asymptotic $=$ line) 


\section{References}

[Chi67] V. P. Chistyakov. Discrete limit distributions in the problem of balls falling in cells with arbitrary probabilities. Math. Notes, 1:6-11, 1967.

[CPSW99] Sylvie Corteel, Boris Pittel, Carla D. Savage, and Herbert S. Wilf. On the multiplicity of parts in a random partition. Random Structures Algorithms, 14(2):185-197, 1999.

[FGD95] Philippe Flajolet, Xavier Gourdon, and Philippe Dumas. Mellin transforms and asymptotics: harmonic sums. Theoret. Comput. Sci., 144(1-2):3-58, 1995. Special volume on mathematical analysis of algorithms.

[FS94] P. Flajolet and R. Sedgewick. Analytic combinatorics - symbolic combinatorics: Saddle point asymptotics. Book in preparation. See also Technical Report 2376, INRIA, 1994.

[GKP89] Ronald L. Graham, Donald E. Knuth, and Oren Patashnik. Concrete mathematics. AddisonWesley Publishing Company Advanced Book Program, Reading, MA, 1989. A foundation for computer science.

[HL01] Paweł Hitczenko and Guy Louchard. Distinctness of compositions of an integer: a probabilistic analysis. Random Structures Algorithms, 19(3-4):407-437, 2001. Analysis of algorithms (Krynica Morska, 2000).

[HRS02] Paweł Hitczenko, Cecil Rousseau, and Carla D. Savage. A generating functionology approach to a problem of Wilf. J. Comput. Appl. Math., 142(1):107-114, 2002. Probabilistic methods in combinatorics and combinatorial optimization.

[HS99] P. Hitczenko and C.D. Savage. On the multiplicity of parts in a random composition of a large integer. Technical report. Avalaible at http://www.csc.ncsu.edu/faculty/savage/, 1999.

[HY97] H.-K. Hwang and Y.-N. Yeh. Measures of distinctness for random partitions and compositions of an integer. Adv. in Appl. Math., 19(3):378-414, 1997.

[JS98] Philippe Jacquet and Wojciech Szpankowski. Analytical de-Poissonization and its applications. Theoret. Comput. Sci., 201(1-2):1-62, 1998.

[Knu73] Donald E. Knuth. The art of computer programming. Volume 3. Addison-Wesley Publishing Co., Reading, Mass.-London-Don Mills, Ont., 1973. Sorting and searching, Addison-Wesley Series in Computer Science and Information Processing.

[Lou87] G. Louchard. Exact and asymptotic distributions in digital and binary search trees. RAIRO Inform. Théor. Appl., 21(4):479-495, 1987.

[LP02] Guy Louchard and Helmut Prodinger. Probabilistic analysis of Carlitz compositions. Discrete Math. Theor. Comput. Sci., 5(1):71-95 (electronic), 2002.

[SČ64] B. A. Sevast'janov and V. P. Čistjakov. Asymptotic normality in the classical problem of balls. Theory of Probability and Applications, 9:198-211, 1964. 